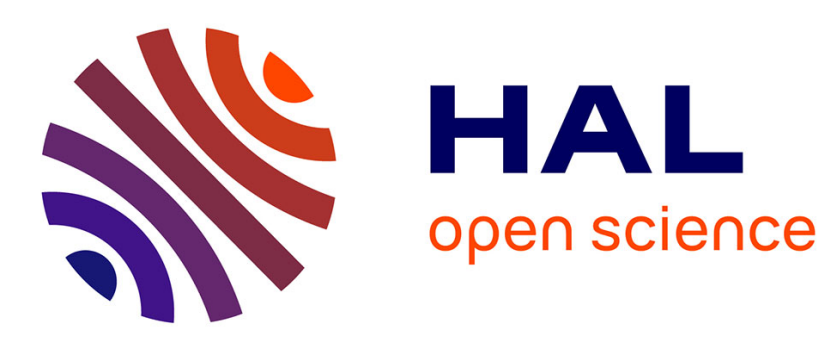

\title{
Random linear multihop relaying in a general field of interferers using spatial Aloha
}

\author{
Bartlomiej Blaszczyszyn, Paul Mühlethaler
}

\section{To cite this version:}

Bartlomiej Blaszczyszyn, Paul Mühlethaler. Random linear multihop relaying in a general field of interferers using spatial Aloha. IEEE Transactions on Wireless Communications, 2015, 17 (7), pp.3700 - 3714. 10.1109/TWC.2015.2409845 . hal-00722000v4

\section{HAL Id: hal-00722000 https://hal.inria.fr/hal-00722000v4}

Submitted on 19 Mar 2015

HAL is a multi-disciplinary open access archive for the deposit and dissemination of scientific research documents, whether they are published or not. The documents may come from teaching and research institutions in France or abroad, or from public or private research centers.
L'archive ouverte pluridisciplinaire HAL, est destinée au dépôt et à la diffusion de documents scientifiques de niveau recherche, publiés ou non, émanant des établissements d'enseignement et de recherche français ou étrangers, des laboratoires publics ou privés. 


\title{
Random linear multihop relaying in a general field of interferers using spatial Aloha
}

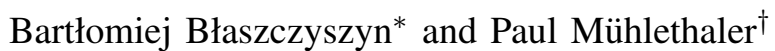 \\ *Inria-ENS, 23 Avenue d'Italie, 75214 Paris, FRANCE; Email: Bartek.Blaszczyszyn@ens.fr \\ †Inria Rocquencourt, Le Chesnay, FRANCE; E-mail: Paul.Muhlethaler@inria.fr
}

\begin{abstract}
In our basic model, we study a stationary Poisson pattern of nodes on a line embedded in an independent planar Poisson field of interfering nodes. Assuming slotted Aloha and the signal-to-interference-and-noise ratio capture condition, with the usual power-law path loss model and Rayleigh fading, we explicitly evaluate several local and end-to-end performance characteristics related to the nearest-neighbor packet relaying on this line, and study their dependence on the model parameters (the density of relaying and interfering nodes, Aloha tuning and the external noise power). Our model can be applied in two cases: the first use is for vehicular ad-hoc networks, where vehicles are randomly located on a straight road. The second use is to study a "typical" route traced in a (general) planar ad-hoc network by some routing mechanism. The approach we have chosen allows us to quantify the non-efficiency of long-distance routing in "pure ad-hoc" networks and evaluate a possible remedy for it in the form of additional "fixed" relaying nodes, called road-side units in a vehicular network. It also allows us to consider a more general field of interfering nodes and study the impact of the clustering of its nodes on the routing performance. As a special case of a field with more clustering than the Poison field, we consider a Poisson-line field of interfering nodes, in which all the nodes are randomly located on random straight lines. In this case our analysis rigorously (in the sense of Palm theory) corresponds to the typical route of this network. The comparison to our basic model reveals a paradox: clustering of interfering nodes decreases the outage probability of a single (typical) transmission on the route, but increases the mean end-to-end delay.
\end{abstract}

Index Terms-MANET, VANET, Aloha, SINR, routing, endto-end delay, Poisson, Poisson-line process, road-side units, clustering, Laplace, comparison.

\section{INTRODUCTION}

The idea of mobile ad-hoc networks (MANETs) - spontaneous wireless networks, operating without a backbone infrastructure, whose users/nodes relay packets for each other in order to enable multihop communication - continues to inspire practitioners and poses interesting theoretical questions regarding its performance capabilities. Vehicular ad-hoc networks (VANETs) may well be currently one of the most promising incarnations of MANETs. Promoters of VANETs believe that these networks will both increase safety on the road and provide value-added services. In order to be able to propose these services, numerous challenging problems must, however, be solved regarding efficient and robust physical layers, reliable and flexible medium access protocols, routing schemes and optimized applications. Using mathematical tools borrowed from stochastic geometry, this paper analyzes the delay of a packet progressing on a linear route, forwarded by randomly positioned nodes using Aloha medium access.

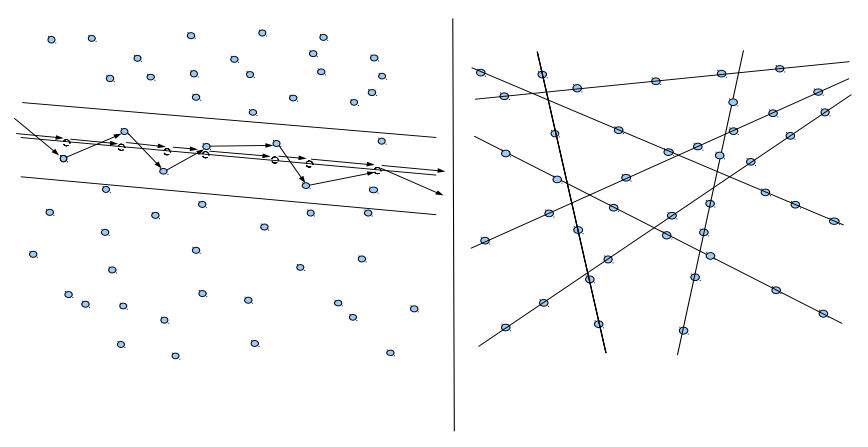

Fig. 1. Left : nodes relaying in given direction and in a given strip approximated by a line of nodes. Right: random nodes on random lines.

The packet transmissions interfere with all the nodes on the line and possibly with an external (non-participating in the routing) field of transmitters, both of which slow down the packet's progression. We believe such a linear network model is adequate for a VANET where vehicles are randomly located on a straight road, and the results established in this paper shed light on VANETs' performance capabilities.

We have also a second, broader, objective which is to contribute to the understanding of the routing performance in planar (2D) MANETs. In the context of a general 2D MANET, the linearity (1D-pattern) of the tagged route may be seen as a simplification. In this case, we can think of packets as being relayed in a given direction (e.g. in a strip or using a geographic routing) as depicted in the left part of Figure 1, with the "real" route approximated by a "virtual" line joining the source and the destination. We will also consider a 2D MANET with all nodes randomly located on random lines, as depicted in Figure 1, on the right. Such a 2D Poissonline MANET model (which can be formalized as a doublystochastic Poisson process, or Cox process) is an interesting alternative to traditional Poisson MANETs. In this case, one tagged route can be rigorously considered as the typical route in this Poisson-line MANET.

\section{A. Route-in-MANET model}

More specifically, we propose an analytically tractable multihop MANET model, by "decoupling" a tagged route from the remaining (external to the route) part of the MANET. In this regard, we consider two stochastically independent point patterns to model respectively: some given route, on which packets of a tagged flow are relayed, and nodes which are only sources of interference for packet transmissions on this 
route. ${ }^{1}$ Moreover, we assume that the tagged route is modeled by a linear (1D) stationary point process. These assumptions allow us to rigorously study rigorously the progress of packets along the route.

Another advantage of the above decoupling is that we can go beyond Poisson assumptions regarding the distribution of nodes both on the route and in the interfering field. In particular, we are able to consider Poisson nodes on the route appended with a periodic (lattice) structure of relaying nodes, which turn out to be crucial to improve routing performance on long routes.

Regarding the external interference, we are able to study the impact of the grouping (clustering) of interfering nodes. In particular, we observe and explain an interesting paradox: more clustering in this pattern decreases the outage probability of a single (typical) transmission on the route, but increases the mean end-to-end delay. The aforementioned 2D Poisson-line MANET (in which all the nodes form independent 1D Poisson processes supported on straight lines themselves forming a Poisson process of lines) is a notable special case of a MANET which clusters nodes more than the usual Poisson MANET. Indeed, it has statistically larger empty regions and more numerous expected groups of points, as will be explained in this paper.

We consider slotted Aloha MAC to be used both by the nodes of the tagged route and of the interfering part of the MANET and the Signal-to-Interference-and-Noise Ratio (SINR) capture/outage condition, with the power-law path loss model and Rayleigh fading. We assume that all the nodes in the MANET are backlogged, i.e., they always have packets to transmit in their buffers. Moreover, we are interested in the (nearest-neighbour) routing performance of a tagged packet relayed by successive nodes on the route with priority in all queues on the route. This latter assumption is particularly appropriate for safety messages, such as Decentralized Environmental Notification Messages (DENMs) propagated in VANETs.

\section{B. Summary of the results}

The main results of this paper are the following:

- In the absence of external (to the route) noise and interference, we evaluate the mean local delay on the Poisson route, i.e. the expected number of time slots required for the packet to be transmitted by the typical node of the route to its nearest neighbor in a given direction. The inverse of this delay is intrinsically related to the speed of packet progression on asymptotically infinite routes, which can also be related to the route transport capacity (number of bit-meters pumped per unit length of the route).

- The mean local delay is minimized (equivalently, the progression speed is maximized) at a unique value of the Aloha medium access probability $p$. Moreover, the routing is unstable - the delay is infinite (the speed

\footnotetext{
${ }^{1}$ In a sense, this is an extension of the well-accepted bipolar MANET model, in which a tagged pair emiter-receiver is considered in the field of interferers; cf. [1, Sec. 16.2.1].
}

is null) - for $p$ larger than some critical value. This observation is fully compliant with the phase-transition phenomenon of the mean local delay discovered in the 2D Poisson MANET model in [2].

- We evaluate the mean end-to-end delay of the packet routing between two given nodes of the route. It shows a cut-off phenomenon, namely that nearest-neighbor routing on distances shorter than some critical value is very inefficient in terms of exploiting the route transport capacity.

- We study the impact of noise and external interference on our previous observations. Confirming the theoretical findings of [3] in 2D Poisson MANETs, we observe that the routing on a Poisson route over long distances is unfeasible: the speed of packet progression on such routes is null due to the existence of overly large hops in random (Poisson) paths. Evaluating the end-to-end delay on finite distances with noise, we identify another, double cut-off phenomenon: the existence of critical values of end-toend distance and noise power, beyond which the speed of packet progression is close to 0 , again making the routing inefficient in terms of exploiting its transport capacity.

- In order to allow efficient routing over long routes one can complete the Poisson route with a fixed lattice of (equidistant) relaying nodes. For this model, we evaluate the mean local delay and show how the route transportcapacity can be maximized by an optimal choice of the inter-relay distance of the lattice structure.

- We explicitly evaluate the Poisson-line MANET model and compare it to the (basic) Poisson-line-in-Poissonfield model. We show that a typical transmission in the Poisson-line model has a smaller outage probability but the routing suffers larger end-to-end delays. This paradox is of a more general nature, and we address it in the light of the theory of clustering comparison of point processes; cf [4-6].

\section{Related works}

An almost ubiquitous stochastic assumption in the theoretical studies of these problems in MANETs is that the nodes of the network are distributed (at any given time) as points of a planar (2D) Poisson point process. In conjunction with the Aloha medium access (MAC) scheme - simpler but less efficient than CSMA which is usually considered in this context by practitioners - 2D Poisson MANET models allow one for quite explicit evaluation of several performance metrics.

Local (one-hop) characteristics in 2D Poisson MANETS, such as SINR outage probability, the related density of packet progress, mean local delay and many others, have been extensively studied in the literature; cf. e.g. among others [2, 712]. Poisson models also allow one to discover some theoretical limitations of macroscopic properties of MANETs, e.g. the scaling of the capacity of dense and/or extended networks $[13,14]$, or the speed of packet progression on long routes $[3,15]$.

Obtaining quantitative, middle-scale, results (e.g. end-to-end routing delays) is however much more difficult. One reason 
for this is that while the source-node can be considered as a typical node in the MANET and the powerful Palm theory of point process can be used in the analysis of the first hop, further relay nodes on a given path (traced by the Dijkstra algorithm or any reasonable local routing on a 2D Poisson MANET) cannot be seen as typical nodes of a MANET. In fact, the route followed by a packet is a random subset of the MANET's point pattern (depending on the routing algorithm) and the typical point "seen" by the packet on a long route is not the typical point of the whole MANET in the sense of Palm theory, unless a high node mobility can be assumed. This would result in completely independent re-sampling of node location after each hop, which might be reasonable in the context of delay-tolerant networks [16], but which we do not want to assume in this paper, cf routing paradox in [1, $\S 21.7 .2 .2]$.

Separating (decoupling) the routing path from the remaining nodes of the network allows us to consider a typical node on the route. More precisely, the packets relayed through nearestneighbor routing on a stationary, linear (1D) pattern of nodes "see", at any relaying node, the typical (1D Palm) distribution of the whole route. This is a well-known point-shift invariance property of the Palm distribution in $1 D$, which does not have a natural extension in higher dimensions.

A very similar scenario is considered in $[17,18]$, where the authors study delay and throughput in a model with relays which are placed equidistantly on the source-destination line. Besides the fact that the topology of relaying nodes is regular (they are equidistant) the authors of this paper assume that the pattern on interfering nodes is re-sampled at each slot (in contrast to our random but static pattern). This major simplification allows them to include both the transmission delays and waiting times in the buffers on the given route (which we ignore). A combined TDMA/ALOHA MAC protocol with intra-route TDMA and inter-route ALOHA is employed. In contrast, we use simple Aloha, ignore queueing, and focus on the performance issues caused by the randomness of the topology of relaying nodes. We are interested in intrinsic limitations of the performance of long-distance routing in wireless networks with an irregular topology, which are not observable in the model with node re-sampling ( $\mathrm{cf}[2]$ ). Comparison of the fixed (correlated) and independent (re-sampled) interference field assumption is also performed in [19], where the joint distribution of the local delays and the mean end-to-end delay on a deterministic route in Poisson fields of interfering nodes is evaluated.

We believe that the limitations observed would remain valid for networks employing CSMA. This is because they primarily depend on the existence of (arbitrarily) long hops. CSMA, which copes better with interference, cannot improve upon this situation. In contrast, we show that using a regular structure of relay nodes superposed with irregular MANET routes leads to better performance. This solution is also evidently necessary for the stability of queuing processes (not covered in our paper).

Regarding VANETs more specifically, [20] considers the packet propagation speed under a protocol (not the physical, based on the SINR) connectivity model, assuming mobile nodes moving in opposite directions. In this (quite different to our) scenario, information can either be propagated at the average speed of the vehicles or much faster, provided the density of vehicles moving in the considered propagation direction is large enough with respect to the density of vehicles moving in the opposite direction. Propagation of information under protocol models is also considered in [21, 22].

The remaining part of this paper is organized as follows. In Section II, we present our nearest neighbor routing model with slotted Aloha. In Section III we compute routing delays in deterministic networks. In Section IV we study the end-toend delays on a Poisson route when the interference is limited to the interfering nodes on the Poisson route. In Section V we study the impact of external noise and interference. Section VI concludes the paper.

\section{LINEAR NEAREST NEIGHBOR ROUTING MODEL IN A SPATIAL MANET WITH SLOTTED ALOHA}

\section{A. A tagged route}

Let us denote by $\mathcal{R}=\left\{X_{i}, i=\ldots,-1,0,1, \ldots\right\}$, with $X_{i} \in \mathbb{R}^{2}$, the locations of nodes participating in the routing of some tagged flow of packets from $X_{i}$ to $X_{i+1}$. This route is assumed not to change on the time scale considered in this paper.

The following assumptions regarding $\mathcal{R}$ will be considered.

1) Deterministic route: Although it is not the main scenario of this paper, we begin by considering a deterministic, fixed, finite pattern of nodes $\mathcal{R}=\left\{X_{0}, \ldots, X_{M}\right\}$.

2) Poisson-line route: In this scenario we suppose that $\mathcal{R}=\Phi$ forms a Poisson point process of intensity $\lambda$, on the line $\mathbb{R}$. In this model the notational convention is such that $X_{i}<X_{i+1}$ and packets are sent by any given node $X_{i}$ to its nearest-to-the-right neighbor $X_{i+1}$. Note that the Poisson assumption means that the 1-hop distances $X_{i+1}-X_{i}$ are independent (across $i$ ) exponential random variables with some given mean $1 / \lambda$. We will call this scenario nearest neighbor $(N N)$ routing on Poisson line. We will also consider a version of this model, where the packet is transmitted to the nearest (available) receiver $(N R)$ to the right on $\mathcal{R}$. This is an opportunistic routing allowing for longer hops, as will be explained in Section II-F. ${ }^{2}$

3) Poisson-line route with fixed relay nodes: In this model, the tagged route consists of a superposition $\mathcal{R}=\Phi \cup \mathcal{G}$, where the Poisson route $\Phi$ is completed with equidistantly located "fixed" relay nodes $\mathcal{G}=\left\{n \Delta+U_{\Delta}, n \in \mathbb{Z}\right\} ; \Delta>0$ is some fixed parameter and $U_{\Delta}$ is a uniform random variable on $[0, \Delta)$, independent of $\Phi$, making $\mathcal{R}$ stationary. In this model we also consider NN routing, i.e., packets are always transmitted to the nearest neighbor to the right in $\mathcal{R}$.

\section{B. A Route in a MANET}

We consider $\mathcal{R}$ as some "tagged" route obtained in a MANET by some routing mechanism. A simple way of extending $\mathcal{R}$ to a $2 \mathrm{D}$ MANET model consists in embedding

\footnotetext{
${ }^{2}$ We implicitly assume that a transceiver is either in a receive (RX) or transmit (TX) mode, but not IDLE, nor in both receive and transmit (RX and TX).
} 
$\mathcal{R}$ in an external field of nodes $\Psi=\left\{Y_{i}\right\}$, on the plane $\mathbb{R}^{2}$. When doing so we will always consider that $\mathcal{R}$ and $\Psi$ are independent.

The following assumptions regarding $\Psi$ will be considered.

1) Fixed pattern of interferers: One may consider a fixed, deterministic pattern $\Psi=\left\{Y_{i}\right\}$, although, once again, it is not the main scenario considered in this paper.

2) Poisson field of interferers: In this model, we assume that $\Psi$ is a Poisson point process of some given intensity $\mu$ on $\mathbb{R}^{2}$. The Poisson linear route $\mathcal{R}=\Phi$ embedded in a Poisson process of interferers will be our default Poisson-linein-Poisson field model.

3) Poisson-line interferers: We will also consider a case where the interferers are located on a Poisson process of lines (roads) on $\mathbb{R}^{2}$ of rate $\nu$ representing the total line-length per unit of surface. Assuming that on each line of this process there is an independent Poisson process of points of intensity $\lambda^{\prime}$ nodes per unit of line length, we obtain a doubly stochastic Poisson point process on $\mathbb{R}^{2}$ with intensity $\mu=\lambda^{\prime} \nu$ nodes per unit of surface; see [23]. In particular, assuming $\lambda=\lambda^{\prime}$, one can rigorously consider the Poisson liner route $\mathcal{R}=\Phi$ embedded in such a Poisson line process of interferers, as the typical route of the Poisson-line MANET, see Figure 1 on the right-hand side.

\section{Aloha MAC}

We assume that all the nodes of $\mathcal{R}$ and $\Psi$ try to access the channel (transmit packets) according to the Aloha scheme, in perfectly synchronized time slots $n=1,2, \ldots$. Each node in each time slot independently tosses a coin with some bias for heads, which will be referred to as the Aloha medium access probability (Aloha MAP). Nodes whose outcome is heads transmit their packets, the others do not transmit. We denote by $p$ the MAP of nodes in $\mathcal{R}$, and by $p^{\prime}$ the MAP of nodes in $\Psi$. The above situation will be modeled by marking the points $X_{i} \in \mathcal{R}$ with random, Bernoulli, medium access indicators $e_{X_{i}}^{n}$ equal to 1 for the nodes which are allowed to emit in the slot $n$ and 0 for the nodes which are not allowed to emit. We have $\mathbf{P}\left(e_{i}^{n}=1\right)=p$ for all $i, n$. When there is no ambiguity, we will skip the time index $n$ in the notation and also use the notation $e_{X_{i}}=e_{i}$. Similarly, we mark the interfering nodes by independent Bernoulli, medium access indicators with parameter $p^{\prime}$.

At each time slot $n$, Aloha splits $\mathcal{R}$ into two point processes $\mathcal{R}^{1}=\mathcal{R}^{1, n}$ of emitters (having a MAC indicator $e$ equal to 1 at time $n$ ) and (potential) receivers $\mathcal{R}^{0}=\mathcal{R}^{0, n}$. It is known that when $\mathcal{R}=\Phi$ is a Poisson process of intensity $\lambda$ then $\mathcal{R}^{1}$ and $\mathcal{R}^{0}$ are independent Poisson processes with intensity $\lambda p$ and $\lambda(1-p)$, respectively.

\section{Signal propagation}

Each transmitting node uses the same transmission power, which without loss of generality is assumed to be equal to $1^{3}$. The signal-power path-loss is modeled by the power-law

\footnotetext{
${ }^{3}$ by measuring external noise in ratio to the actually transmitted power
}

function $l(r)=(A r)^{\beta}$ where $A, \beta>0$ are some constants and $r$ is the distance between the transmitter and the receiver.

Signal-power is also perturbed by random fading $F$ which is independently sampled for each transmitter-receiver pair at each time slot $n$. Thus, the actual signal-power received at $y$ from $x$ at time $n$ is equal to $F_{(x, y)}^{n} / l(|x-y|)$. In this paper we will restrict ourselves to an important special case where $F$ is exponentially distributed, which corresponds to the situation of independent Rayleigh fading. By renormalization of $A$, if required, we can assume without loss of generality that $F$ has mean 1 .

\section{E. SINR capture}

When a node located at $x$ transmits a signal to a node located at $y$, then successful reception depends on the signalto-interference-and-noise ratio (SINR)

$$
\operatorname{SINR}_{(x, y)}=\operatorname{SINR}_{(x, y)}^{n}=\frac{F_{(x, y)}^{n} / l(|x-y|)}{W^{n}(y)+I_{\mathcal{R}^{1, n} \backslash\{x\}}(y)},
$$

where $I_{\mathcal{R}^{1, n} \backslash\{x\}}$ is the shot-noise process of $\mathcal{R}^{1, n}$ from which transmitter $x$ is subtracted: $I_{\mathcal{R}^{1, n} \backslash\{x\}}(y)=$ $\sum_{X_{i} \in \mathcal{R}^{1, n} \backslash\{x\}} F_{\left(y, X_{i}\right)}^{n} / l\left(\left|y-X_{i}\right|\right)$ representing the interference created by the nodes of route $\mathcal{R}$ and $W^{n}(y)$ represents an external (to $\mathcal{R}$ ) noise (normalized by the actual transmission power, cf footnote 3 ). This external noise can be a constant ambient noise $W^{n}(y)=W$ or a random field. In particular, $W^{n}(y)$ may comprise the interference created by the external field of interferers $\Psi$, which do not belong to the route $\mathcal{R}$. In this case:

$$
W^{n}(y)=W+I_{\Psi^{1, n}}(y)=W+\sum_{Y_{i} \in \Psi^{1, n}} F_{\left(y, Y_{i}\right)}^{n} / l\left(\left|y-Y_{i}\right|\right),
$$

where $\Psi^{1, n}$ is the subset of nodes of $\Psi$ transmitting at time $n$. Throughout the paper we assume that $W, \Psi$ and $\mathcal{R}$ are mutually independent, with independent MAC decisions and fading variables.

In this paper we assume a fixed bit-rate coding, i.e., $y$ successfully receives the signal from $x$ if

$$
\operatorname{SINR}_{(x, y)} \geq T,
$$

where $\operatorname{SINR}_{(x, y)}$ is given by (2.1) and $T$ is the SINR-threshold related to the bit-rate given some particular coding scheme.

\section{F. NN versus $N R$ routing}

Recall that in our NN routing model each transmitter in $\mathcal{R}^{1}$ transmits to the nearest node on its right in $\mathcal{R}$, without knowing whether it is authorized to transmit at this time. Successful reception requires that this selected node is not authorized by Aloha to transmit (i.e., it is in $\mathcal{R}^{0}$ ), and that the SINR condition (2.3) for this transmitter-receiver pair is satisfied. This corresponds to the usual separation of the routing and MAC layers. In contrast, NR routing consists in transmitting to the nearest node (in the given direction) on $\mathcal{R}$ which, at the given time slot, is not authorized by Aloha to transmit. The NR model hence might be seen as an opportunistic routing, which requires some interplay between 
MAC and the routing layer. As we shall see, both routing schemes allow one for quite explicit performance analysis in our basic Poisson-line-in-Poisson-field model.

\section{G. Numerical assumptions}

The default assumption in our numerical examples throughout the paper is $\lambda=0.01$ nodes per meter in the Poisson line model, i.e. the mean distance between two consecutive nodes on the line is $100 \mathrm{~m}$. We will express all (transmission) delay times in seconds (s) and the speed (of packet progression) in meters per slot duration.

We will also use $A=1, \beta=4$ and $T=10$. The fading $F$ is Rayleigh, $F$ is exponentially distributed with mean 1, $\mathbf{E}(F)=1$.

\section{PRELIMINARIES: CALCULUS OF ROUTING DELAYS IN DETERMINISTIC NETWORKS}

Unless otherwise specified, in this section we assume that the locations of the nodes in the network are known and fixed (deterministic). The only source of randomness is Aloha MAC and independent Rayleigh fading between any two given nodes. In what follows we present a simple computation which allows us to express coverage and routing delays in such networks. Our observations will be useful in the remaining part of this paper, when we will study random routes in random MANETs.

1) Coverage probability: Let us consider a transmitter located at $x \in \mathbb{R}^{2}$ communicating to a receiver located at $y \in \mathbb{R}^{2}$ in the presence of a field $\Psi=\left\{y_{i} \in \mathbb{R}^{2}\right\}$ of interferers, with all nodes subject to Aloha MAC. For the sake of generality we assume that $x$ and $y$ use Aloha with MAP $p$, while the nodes in $\Psi$ use a (possibly different) MAP $p^{\prime}$. We assume that $x, y, \notin \Psi$. We denote by $\Pi(x, y, \Psi)$ the probability of successful transmission from $x$ to $y$ in one time slot. This probability accounts for the likelihood of $x$ being authorized by Aloha to transmit, $y$ not to being authorized to transmit and, given such circumstances, the probability of achieving the SINR larger than $T$ at the receiver.

We define the following functions:

$$
\begin{gathered}
h(s, r)=1-\frac{p}{\frac{1}{T}(s / r)^{\beta}+1} \quad s, r \geq 0, \\
w(s)=\exp \left(-T W(A s)^{\beta}\right) \quad s \geq 0 .
\end{gathered}
$$

Also, we will write $h^{\prime}(s, r)$ for the function given by (3.4) with $p$ replaced by $p^{\prime}$. For reasons which will become clear in what follows, we call $h(s, r)$ the interference factor and $w$ the noise factor.

Lemma 3.1: We have

$$
\Pi(x, y, \Psi)=p(1-p) w(|x-y|) \prod_{z \in \Psi} h^{\prime}(|z-y|,|x-y|) .
$$

Remark 3.2: Let us note that $\Pi$ satisfies the following recursion when adding an interferer $z$ to the field $\Psi$ :

$$
\begin{aligned}
\Pi(x, y, \emptyset) & =p(1-p) w(|x-y|), \\
\Pi(x, y, \Psi \cup\{z\}) & =\Pi(x, y, \Psi) h^{\prime}(|z-y|,|x-y|) .
\end{aligned}
$$

In other words, the external noise reduces the probability of successful transmission over the distance $r$ by the factor $w(r)$, which in the absence of noise and interference is equal to $p(1-p)$ (because of the Aloha scheme). Moreover, adding an interfering node $z$ to $\Psi$ causes a decrease in the successful transmission probability by the factor $h^{\prime}(s, r)$, where $s$ is the distance of the new interferer to the receiver.

Proof of Lemma 3.1: We have

$$
\begin{aligned}
& \Pi(x, y,\{z\}) \\
& =p(1-p) \mathbf{P}\left\{F_{(x, y)} / l(|x-y|) \geq T\left(W+e_{z} F_{(z, y)} / l(|y-z|)\right\}\right. \\
& =p(1-p) e^{-T W l(|x-y|)} \mathbf{E}\left[e^{-T e_{z} F_{(z, y)} l(|x-y|) / l(|z-y|)}\right]
\end{aligned}
$$

and

$$
\begin{aligned}
& \mathbf{E}\left[e^{-T e_{x} F_{(z, y)} l(|x-y|) / l(|z-y|)}\right] \\
& =\left(1-p^{\prime}\right)+p^{\prime} \mathbf{E}\left[e^{-T F_{(z, y)} l(|x-y|) / l(|z-y|)}\right] \\
& =1-\frac{p^{\prime}}{\frac{1}{T} \frac{|z-y|^{\beta}}{|x-y|^{\beta}}+1},
\end{aligned}
$$

where we use the assumption that $F$ is an exponential random variable. The proof follows by induction.

\section{A. Local routing delay}

$\Pi(x, y, \Psi)$ is the probability that node $x$ can successfully send a tagged packet to node $y$ in a single transmission. However, a single transmission is not sufficient in Aloha. Hence, after an unsuccessful transmission, the transmitter will try to retransmit the packet with Aloha, possibly several times, until the packet's reception. We denote by $L(x, y, \Psi)$ the expected number of time slots required to successfully transmit a packet from $x$ to $y$ (considering the previous scenario of $x \in \mathbb{R}^{2}$ transmitting to $y \in \mathbb{R}^{2}$ in the presence of a field $\Psi=\left\{y_{i} \in \mathbb{R}^{2}\right\}$ of interferers). Under our assumptions this number is a geometric random variable of parameter $\Pi(x, y, \Psi)$ and hence its expected value is equal to:

$$
L(x, y, \Psi):=\frac{1}{\Pi(x, y, \Psi)} .
$$

We call $L(x, y, \Psi)$ the local (routing) delay. By Lemma 3.1 we have immediately the following result.

\section{Lemma 3.3:}

$L(x, y, \Psi)=\frac{1}{p(1-p)} w^{-1}(|x-y|) \prod_{z \in \Psi} h^{\prime-1}(|z-y|,|x-y|)$.

and an analogous recurrence to this of Remark 3.2 holds for $L$ with the reciprocals of the noise and interference factors.

\section{B. Route delays}

Let us now consider a route consisting of nodes $\mathcal{R}=$ $\left\{x_{0}, x_{1}, \ldots, x_{n}\right\}$ in the field of interfering nodes $\Psi$ (we assume that $\mathcal{R} \cap \Psi=\emptyset$ ). All nodes in $\mathcal{R}$ use MAP $p$ while interferers in $\Psi$ (which are considered as "external"to the route) use MAP $p^{\prime}$. The average delay to send a packet from $x_{0}$ to $x_{n}$ using the route $\mathcal{R}$ is simply the sum of the local 
delays on successive hops

$$
L(\mathcal{R}, \Psi):=\sum_{k=0}^{n-1} L\left(x_{k}, x_{k+1}, \Psi \cup \mathcal{R} \backslash\left\{x_{k}, x_{k+1}\right\}\right) .
$$

Note that for the hop from $x_{k}$ to $x_{k+1}$ other nodes of the route $\mathcal{R} \backslash\left\{x_{k}, x_{k+1}\right\}$ act as interferers. We call $L(\mathcal{R}, \Psi)$ the (routing) delay on $\mathcal{R} .{ }^{4}$ Using (3.11) we obtain

$$
\begin{aligned}
L(\mathcal{R}, \Psi)= & \frac{1}{p(1-p)} \sum_{k=0}^{n-1} w^{-1}\left(\left|x_{k}-x_{k+1}\right|\right) \\
& \times \prod_{z \in \mathcal{R} \backslash\left\{x_{k}, x_{k+1}\right\}} h^{-1}\left(\left|z-x_{k+1}\right|,\left|x_{k}-x_{k+1}\right|\right) \\
& \times \prod_{z \in \Psi} h^{\prime-1}\left(\left|z-x_{k+1}\right|,\left|x_{k}-x_{k+1}\right|\right) .
\end{aligned}
$$

We also denote by

$$
V(\mathcal{R}, \Psi):=\frac{\left|x_{n}-x_{0}\right|}{L(\mathcal{R}, \Psi)}
$$

the mean speed of packet progression on the route $\mathcal{R}$.

\section{Introducing stochastic geometry}

The above expressions allow one for a relatively simple, explicit analysis of routing delays in fixed networks, i.e. in networks where the locations of the nodes are fixed and known. In the case when such information is not available, one adopts a stochastic-geometric approach averaging over possible geometric scenarios regarding $\mathcal{R}$ and/or $\Psi$. For example, assume that the route $\mathcal{R}$ is given and fixed, but the number and precise locations of interferers are not given. Assuming some statistical hypothesis regarding the distribution of $\Psi$ (which becomes random point process) one can calculate the average route delay $\mathbf{E}_{\Psi}[L(\mathcal{R}, \Psi)]$ where the expectation regards the distribution of $\Psi$. Specifically, for a given couple $(x, y) \in \mathbb{R}^{2}$ we define a function

$$
H_{x, y}(z):=\log (h(|z-y|,|x-y|)), \quad z \in \mathbb{R}^{2}
$$

and similarly $H_{x, y}^{\prime}(z)$ for $h(\cdot, \cdot)$ replaced by $h^{\prime}(\cdot, \cdot)$. We denote by $\mathcal{L}_{\Psi}$ the Laplace transform of $\Psi$, i.e., for a given (say non-negative) function $f(\cdot)$ defined on $\mathbb{R}^{2}, \mathcal{L}_{\Psi}(f):=$ $\mathbf{E}_{\Psi}\left[\exp \left(-\sum_{Y_{i} \in \Psi} f\left(Y_{i}\right)\right)\right]$. We then have:

$$
\begin{aligned}
& \mathbf{E}_{\Psi}[\Pi(x, y, \Psi)]=p(1-p) w(|x-y|) \mathcal{L}_{\Psi}\left(-H_{x, y}^{\prime}\right), \\
& \mathbf{E}_{\Psi}[L(x, y, \Psi)]=\frac{1}{p(1-p)} w^{-1}(|x-y|) \mathcal{L}_{\Psi}\left(H_{x, y}^{\prime}\right)
\end{aligned}
$$

and

$$
\begin{aligned}
\mathbf{E}_{\Psi}[L(\mathcal{R}, \Psi)]= & \frac{1}{p(1-p)} \sum_{k=0}^{n-1} w^{-1}\left(\left|x_{k}-x_{k+1}\right|\right) \mathcal{L}_{\Psi}\left(-H_{x_{k}, x_{k+1}}^{\prime}\right) \\
& \times \prod_{z \in \mathcal{R} \backslash\left\{x_{k}, x_{k+1}\right\}} h^{-1}\left(\left|z-x_{k+1}\right|,\left|x_{k}-x_{k+1}\right|\right) .
\end{aligned}
$$

In Section IV we will consider a scenario when the route $\mathcal{R}$ is also modeled as a point process. Before this, let us study

\footnotetext{
${ }^{4}$ Observe that the routing delay does not take into account packet queuing at the relay nodes.
}

the impact of the structure of the interfering points process on the transmission between two given points.

\section{More versus less clustering for the interferers}

In order to simplify the notation let us denote $\mathcal{L}_{\Psi}^{-H^{\prime}}(\mid x-$ $y \mid):=\mathcal{L}_{\Psi}\left(-H_{x, y}^{\prime}\right)$ and similarly $\mathcal{L}_{\Psi}^{H^{\prime}}(|x-y|):=\mathcal{L}_{\Psi}\left(H_{x, y}^{\prime}\right)$. In what follows we will consider two particular models for $\Psi$ : Poisson point process (P) of intensity $\mu$ (cf Section II-B2) and Poisson-line process (PL) of line intensity $\nu$ and points-online intensity $\lambda$ (cf Section II-B3). For these two processes we denote $\mathcal{L}_{\Psi}^{-H^{\prime}}(r)$ and $\mathcal{L}_{\Psi}^{H^{\prime}}(r)$ respectively by $\mathcal{L}_{P}^{-H^{\prime}}(r), \mathcal{L}_{P}^{H^{\prime}}(r)$ and $\mathcal{L}_{P L}^{-H^{\dagger}}(r), \mathcal{L}_{P L}^{H^{\prime}}(r)$.

Lemma 3.4: For the Poisson model of interferers $\Psi$ we have:

$$
\begin{aligned}
\mathcal{L}_{P}^{-H^{\prime}}(r) & =\exp \left(-\frac{2 \pi^{2} \mu p^{\prime} r^{2} T^{2 / \beta}}{\beta \sin (2 \pi / \beta)}\right), \\
\mathcal{L}_{P}^{H^{\prime}}(r) & =\exp \left(\frac{2 \pi^{2} \mu p^{\prime} r^{2} T^{2 / \beta}}{\beta\left(1-p^{\prime}\right)^{1-2 / \beta} \sin (2 \pi / \beta)}\right) .
\end{aligned}
$$

For the Poisson-line interferers we have:

$$
\begin{aligned}
& \mathcal{L}_{P L}^{-H^{\prime}}(r)=\exp \left(-2 \nu r T^{1 / \beta}\right. \\
& \left.\times \int_{0}^{\infty}\left(1-\exp \left(-2 \lambda^{\prime} r T^{1 / \beta} p^{\prime} \int_{0}^{\infty} \frac{1}{\left(s^{2}+t^{2}\right)^{\beta / 2}+1} \mathrm{~d} t\right)\right) \mathrm{d} s\right) \\
& \mathcal{L}_{P L}^{H^{\prime}}(r)=\exp \left(-2 \nu r T^{1 / \beta}\right. \\
& \left.\int_{0}^{\infty}\left(1-\exp \left(2 \lambda^{\prime} r T^{1 / \beta} p^{\prime} \int_{0}^{\infty} \frac{1}{\left(s^{2}+t^{2}\right)^{\beta / 2}+1-p^{\prime}} \mathrm{d} t\right)\right) \mathrm{d} s\right)
\end{aligned}
$$

Proof: All the expressions follow from the evaluation of the Laplace transforms of the respective point processes. For a radially symmetric function $f$ we have the following well known expression for the Laplace transform of the Poisson process $\Psi$ of intensity $\mu$

$$
\mathcal{L}_{\Psi}(f)=\mathcal{L}_{P}(f)=\exp \left(-2 \pi \mu \int_{0}^{\infty} 1-\exp (-f(s)) s \mathrm{~d} s\right) .
$$

Expressions (3.16) and (3.17) are known in the literature, cf $[1,(16.18)$ and (17.34) in the context of the preceding equation ibid.], respectively. The Laplace transform of the Poisson-line field of interferers can be found in [23]

$$
\begin{aligned}
& \mathcal{L}_{\Psi}(f)=\mathcal{L}_{P L}(f) \\
& =\exp \left(-2 \nu \int_{0}^{\infty} 1-e^{-2 \lambda^{\prime} \int_{0}^{\infty} 1-\exp \left(-f\left(\sqrt{s^{2}+t^{2}}\right)\right) \mathrm{d} t} \mathrm{~d} s\right)
\end{aligned}
$$

Corollary 3.5: (Interferers' clustering paradox) We have the following inequalities $\mathcal{L}_{P L}^{-H^{\prime}}(r) \geq \mathcal{L}_{P}^{-H^{\prime}}(r)$ and $\mathcal{L}_{P L}^{H^{\prime}}(r) \geq$ $\mathcal{L}_{P}^{H^{\prime}}(r)$ for all $r \geq 0$, provided $\mu=\lambda^{\prime} \nu$. In consequence, both the probability of successful transmission $\Pi(x, y, \Psi)$ and the local routing delay $L(x, y, \Psi)$ are larger in the model with the Poisson-line field of interferers than with the homogeneous Poisson field having the same spatial intensities of points. 
The above result states that grouping interfering nodes in clusters (on lines, in the Poisson-line model) is beneficial for the transmission probability but harmful for the end-toend delays. This apparent paradox can be explained in the following way. Clustered interfering nodes leaves statistically larger "vacant" regions and thus increases the probability that a given transmission from $x$ to $y$ takes place in such a region. However, when this transmission falls within a region where interfering nodes have higher density, the local delay increases considerably and actually makes the average delay larger even if the probability of this event (falling within a region with a high density of interferers) is smaller.

Remark 3.6: The result of Corollary 3.5 extends to arbitrary two point processes of interferers $\Psi$, which are comparable in the sense of Laplace transforms of both positive and negative functions. More precisely, the capture probability is expressed as the Laplace transform of a positive function $\left(-H_{x, y}^{\prime} \geq 0\right)$, while the end-to-end delay requires the transform of a negative function $\left(H_{x, y}^{\prime} \leq 0\right)$. The ordering of Laplace transforms of point processes in the positive domain is equivalent to the ordering of void probabilities while the ordering of all moment measures implies the Laplace transform ordering in the negative domain. It is quite natural to consider both cases as complementary ways of comparing clustering properties of point processes. In general, Poisson-Poisson cluster point processes (to which our Poisson-line MANET belongs) cluster nodes more than a homogeneous Poisson process; cf [5] and also [6] for more details and numerous examples.

Proof of Corollary 3.5: The result follows from the comparison of the respective Laplace transforms of Poisson and Poisson-line processes of interferers. This can be concluded from a more general theory of clustering comparison of point processes, noting that our Poisson-line processes of interferers is in fact an instance of a Poisson-Poisson cluster process, which is a super-Poisson ( $d c x$-larger than Poisson) process, cf [6, Example 16]. The following direct comparison of the respective Laplace transforms confirms this general observation. Using the inequality $e^{x} \geq 1+x$ for all $x$ and (3.21) we have:

$$
\begin{aligned}
& \mathcal{L}_{P L}(f) \\
& \geq \exp \left(-4 \nu \lambda^{\prime} \int_{0}^{\infty} \int_{0}^{\infty} 1-\exp \left(-f\left(\sqrt{s^{2}+t^{2}}\right)\right) \mathrm{d} t \mathrm{~d} s\right) \\
& \geq \exp \left(-2 \pi \nu \lambda^{\prime} \int_{0}^{\infty} 1-\exp (-f(s)) s \mathrm{~d} s\right),
\end{aligned}
$$

with the last expression giving the Laplace transform of the Poisson point process of intensity $\nu \lambda^{\prime}$ applied to radially symmetric function $f$. This completes the proof.

\section{END-TO-END DELAYS ON A POISSON ROUTE IN THE ROUTE-INTERFERENCE LIMITED CASE}

In this section we assume Poisson route $\mathcal{R}=\Phi$ and no external interferers $(\Psi \equiv \emptyset)$. We also assume that the external noise process $W^{n}(y) \equiv 0$ is negligible with respect to the interference created by the nodes participating in the routing. In this scenario we will consider NN and NR routing schemes; cf II-A2. We begin with a simple calculation of the capture probability.

\section{A. Capture probability}

We consider a typical node on the route $\mathcal{R}$, that is of the Poisson point process $\Phi$. By the Slivnyak theorem, it can be seen as an "extra" node located at the origin $X_{0}=0$, with the other nodes of the route distributed according to the original stationary Poisson process $\Phi$. All marks (Bernoulli MAC indicators, fading, etc) of these extra nodes are independent of the marks of the points of $\Phi$. We denote by $\Pi_{N N}(p)$ and $\Pi_{N R}(p)$ the probability of successful transmission of the typical node $X_{0}=0$ in a given time slot to the receiver prescribed by the NN and NR routing schemes, respectively. In the capture probability $P_{N N}(p)$ and $P_{N R}(p)$ in the two routing schemes considered, we assume that the typical node is authorized to transmit. Obviously $\Pi_{N N}(p)=p P_{N N}(p)$ and $\Pi_{N R}(p)=p P_{N R}(p)$.

For arbitrary $a, b>0$ we denote $C(a, b)=\int_{a}^{\infty} 1 /\left(u^{b}+\right.$ 1) $\mathrm{d} u$ and

$$
C(b)=C(0, b)=\frac{\pi}{b \sin (\pi / b)} .
$$

Moreover, for given $T$ and $\beta$ let us denote:

$$
\begin{aligned}
& \mathcal{C}_{1}=\mathcal{C}_{1}(T, \beta)=T^{1 / \beta}\left(C\left(T^{-1 / \beta}, \beta\right)+C(\beta)\right) \\
& \mathcal{C}_{2}=\mathcal{C}_{2}(T, \beta)=2 T^{1 / \beta} C(\beta)=\frac{2 T^{1 / \beta} \pi}{\beta \sin (\pi / \beta)} .
\end{aligned}
$$

Proposition 4.1: The probability of successful transmission by a typical node of a Poisson route $\mathcal{R}=\Phi$ authorized by Aloha to transmit to its relay node in the NN routing model without noise is equal to:

$$
P_{N N}(p)=\frac{1-p}{1+p \mathcal{C}_{1}}
$$

and similarly in the $N R$ receiver model

$$
P_{N R}(p)=\frac{1-p}{1+p\left(\mathcal{C}_{2}-1\right)} .
$$

Remark 4.2: It is easy to see that $\mathcal{C}_{2}-1 \leq \mathcal{C}_{1}$ and hence $P_{N R} \geq P_{N N}$, i.e., the opportunistic choice of the receiver pays off regarding the probability of successful transmission.

Proof of Prop. 4.1: Note directly from the form of the SINR in (2.1) that $P_{N N}(p)$ and $P_{N R}(p)$ do not depend on $A$. Hence in the remaining part of the proof we take $A=1$.

We consider first the NN model. The scenario with the typical user $X_{0}=0$ located at the origin corresponds to the Palm distribution $\mathbf{P}^{0}$ of the Poisson route $\mathcal{R}=\Phi \cup\{0\}$. By the known property of the Poisson point process, the distance $R$ from $X_{0}$ to its nearest neighbor to the right, $X_{1}$, has an exponential distribution with parameter $\lambda$. Moreover given $R=r$, all other nodes of the Poisson route $\mathcal{R}$ form a Poisson point process of intensity $\lambda$ on $(-\infty, 0) \cup(r, \infty)$. Conditioning on $R=r$ we can thus use (3.13) with $\Psi=$ $\Phi^{1} \cap((-\infty, 0) \cup(r, \infty))=: \Phi_{r}^{1}$ (recall that $\Phi^{1}$ is a Poisson process of intensity $\lambda p$ of nodes transmitting in a given time 
slot) to obtain:

$$
\begin{aligned}
P_{N N}(p) & =\frac{\lambda}{p} \int_{0}^{\infty} e^{-\lambda r} \mathbf{E}^{0}\left[\Pi\left(0, r, \Phi_{r}^{1}\right)\right] \mathrm{d} r \\
& =\lambda(1-p) \int_{0}^{\infty} e^{-\lambda r} \mathcal{L}_{\Phi_{r}^{1}}\left(H_{0, r}\right) \mathrm{d} r .
\end{aligned}
$$

Using the well known formula for the Laplace transform of the Poisson p.p. (see e.g., [1, (16.4)]) we obtain:

$$
\begin{aligned}
\mathcal{L}_{\Phi_{r}^{1}}\left(H_{0, r}\right) & =\exp \left\{-\lambda p \int_{(-\infty, 0) \cup(r, \infty)}\left(1-e^{H_{0, r}(|s|)}\right) \mathrm{d} s\right\} \\
& =\exp \left\{-\lambda p \int_{(-\infty, 0) \cup(r, \infty)}(1-h(|s|, r)) \mathrm{d} s\right\} \\
& =\exp \left\{-\lambda p \int_{(-\infty, 0) \cup(r, \infty)}\left(\frac{1}{1+(|s| / r)^{\beta} / T}\right) \mathrm{d} s\right\} \\
& =\exp \left\{\lambda p r T^{1 / \beta}\left(C\left(T^{-1 / \beta}, \beta\right)+C(\beta)\right)\right\} .
\end{aligned}
$$

Inserting this expression in (4.27) we obtain

$P_{N N}(p)=\lambda(1-p) \int_{0}^{\infty} e^{-\lambda r\left(1+p T^{1 / \beta}\left(C\left(T^{-1 / \beta}, \beta\right)+C(\beta)\right)\right)} \mathrm{d} r$,

which boils down to the right-hand side of (4.25).

In order to obtain expression (4.26) for the $N R$ model, we follow the same process, with the following modifications. The distance to the nearest receiver to the right has the exponential distribution of parameter $\lambda(1-p)$. Moreover the distribution of the point process of emitters is Poisson with parameter $\lambda p$ and independent of the location of this receiver. Consequently

$$
\begin{aligned}
P_{N R}(p) & =\frac{\lambda(1-p)}{p} \int_{0}^{\infty} e^{-\lambda(1-p) r} \mathbf{E}^{0}\left[\Pi\left(0, r, \Phi^{1}\right)\right] \mathrm{d} r \\
& =\lambda(1-p) \int_{0}^{\infty} e^{-\lambda(1-p) r} \mathcal{L}_{\Phi^{1}}\left(H_{0, r}\right) \mathrm{d} r .
\end{aligned}
$$

Note also that by the very choice of the receiver, it is not authorized to emit in the given time slot, hence there is no extra $(1-p)$ factor in the numerator of (4.29). We have $\mathcal{L}_{\Phi^{1}}\left(H_{0, r}\right)=\exp \left\{\lambda p r T^{1 / \beta} 2 C(\beta)\right\}$ and plugging into (4.29) one obtains (4.26), which completes the proof.

\section{B. Local delays}

Let us denote by $L_{0}$ the local delay at the tagged node in the NN routing scheme, i.e. the number of consecutive time slots needed for the tagged node to successfully transmit a given packet to the receiver designated by the given routing scheme. Local delays will be consider only for NN routing ${ }^{5}$. In what follows we will give the expressions for the expected local delay $\mathbf{E}^{0}\left[L_{0}\right]$ at the typical node of the Poisson route, which we call the average local delay, where the averaging regards all possible Poisson configurations of other nodes $\Phi$. As we will see, this expectation is finite only if $p$ is sufficiently small (for any given $T$ and $\beta$ ).

\footnotetext{
${ }^{5}$ The nearest receiver is not the same across different slots. This makes the calculation of the local delay (local exit time) in NR routing less tractable, and it is omitted in the paper due to space constraints.
}

Let us denote

$$
\begin{aligned}
& \mathcal{D}_{1}(p)=\mathcal{D}_{1}(p ; T, \beta) \\
& \quad=T^{1 / \beta}\left(\int_{T^{-1 / \beta}}^{\infty} \frac{1}{u^{\beta}+1-p} \mathrm{~d} u+\int_{0}^{\infty} \frac{1}{u^{\beta}+1-p} \mathrm{~d} u\right) .
\end{aligned}
$$

Proposition 4.3: Under the assumptions of Proposition 4.1, the mean local delay in the $N N$ routing model is equal to

$$
\mathbf{E}^{0}\left[L_{0}\right]=\frac{1}{p(1-p)\left(1-p \mathcal{D}_{1}(p)\right)}
$$

provided

$$
p \mathcal{D}_{1}(p)<1
$$

and $\bar{L}_{N N}=\infty$ otherwise.

Proof: Conditioning on the distance to the nearest neighbor as in the proof of Proposition 4.1 and using (3.14), we have:

$$
\mathbf{E}^{0}\left[L_{0}\right]=\frac{\lambda}{p(1-p)} \int_{0}^{\infty} e^{-\lambda r} \mathcal{L}_{\Phi_{r}^{1}}\left(-H_{0, r}\right) \mathrm{d} r .
$$

with

$$
\begin{aligned}
\mathcal{L}_{\Phi_{r}}\left(-H_{0, r}\right) & =\exp \left\{-\lambda p \int_{(-\infty, 0) \cup(r, \infty)}\left(1-e^{-H_{0, r}(s)}\right) \mathrm{d} s\right\} \\
& =\exp \left\{-\lambda p \int_{(-\infty, 0) \cup(r, \infty)}\left(1-h^{-1}(s, r)\right) \mathrm{d} s\right\} \\
& =\exp \left\{\lambda p r \mathcal{D}_{1}(p)\right\} .
\end{aligned}
$$

Consequently, we have :

$$
\mathbf{E}^{0}\left[L_{0}\right]=\frac{\lambda}{p(1-p)} \int_{0}^{\infty} e^{-\lambda r\left(1-p \mathcal{D}_{1}(p)\right)} \mathrm{d} r .
$$

It is easy to see that this integral is infinite if $p \mathcal{D}_{1}(p) \geq 1$ and $1 /\left((1-p)\left(1-p \mathcal{D}_{1}(p)\right)\right)$ otherwise. This completes the proof.

Remark 4.4: Similar phase transition (existence of the critical $p$ ) for the mean local delay was discovered in 2D MANETs in [2]. Note that $\mathcal{D}_{1}(p) \geq \mathcal{C}_{1}$ with the equality attained when $p \rightarrow 0$. Hence, for small $p$ we have $\mathcal{D}_{1}(p) \approx \mathcal{C}_{1}$, and the critical $p$ for which the average expected delay explodes is approximately $1 / \mathcal{C}_{1}$.

Expression (4.31) can be used to tune $p$ to the value which minimizes the mean local delay. In the next section we will see that this value of $p$ also optimizes the performance of $N N$ routing on long routes, for almost any realization of the Poisson route.

\section{Long-distance speed of packet progression}

We call long-distance speed the speed at which a packet progresses over a long distance. Let us denote by $D_{i}, i=1, \ldots$ the local delay of the packet on its hop from $X_{i}$ to $X_{i+1}$. More specifically, then $\sum_{i=0}^{k-1} D_{i}$ is the end-to-end delay on $k$ hops on the route that starts at $X_{0}=0$. Recall that we assume that there is no queueing delay for the packet on its path. We denote by $v=\lim _{k \rightarrow \infty}\left|X_{k}\right| / \sum_{i=0}^{k} D_{i}$ the long-distance speed of the packet progression (expressed in the units of distance per slot duration). Note also that $v$ multiplied by the number of bits carried by one packet corresponds to the route transport 
capacity defined as the number of bit-meters "pumped" by the network per slot.

Proposition 4.5: Under the assumptions of Proposition 4.1, the mean long-distance speed of the transmission of packets in $N N$ routing model is equal to:

$$
v=\frac{p(1-p)\left(1-p \mathcal{D}_{1}(p)\right)}{\lambda}
$$

provided $p \mathcal{D}_{1}(p)<1$ and 0 otherwise.

Proof: The mean empirical speed during the first $k$ hops is:

$$
\frac{X_{k}}{\sum_{i=0}^{k-1} D_{i}}=\frac{\left(\sum_{i=0}^{k-1}\left(X_{i+1}-X_{i}\right)\right) / k}{\left(\sum_{i=0}^{k-1} D_{i}\right) / k} .
$$

When $k$ tends to $\infty$ the numerator tends almost surely to $1 / \lambda$. In the case of Poisson (or, more generally, a renewal process) this follows from the classical ergodic theorem for i.i.d. random variables. The denominator tends almost surely to $\mathbf{E}^{0}\left[L_{0}\right]$, which is a consequence of the ergodic theorem for marked ergodic point processes (in our case independently marked Poisson point process); cf e.g. [24, (13.4.1 2)].

Example 4.6: Figure 2 shows the mean long-distance speed for $\lambda=0.01, \beta=4$ and $T=10$. We observe that there is an optimal value of $p$ which maximizes the long-distance speed of packet progression and that this speed drops to 0 for $p$ larger than some critical value. Recall that, in terms of VANETs, $\lambda=0.01$ means that the averge distance between cars is $100 \mathrm{~m}$. If we assume that the slot duration is $1 \mathrm{~ms}$, the mean long-distance speed is $6 \mathrm{~km} / \mathrm{s}$. An emergency packet will take $0.166 \mathrm{~s}$ to propagate over $1 \mathrm{~km}$ which is approximately twice the period generally used for Decentralized Environmental Notification Messages (DENMs) i.e. 100ms.

Remark 4.7: Existence of the critical value of $p$ can be attributed to hops, traversed by a tagged packet on the infinite route, being statistically too long. Indeed, analysing the expression in (4.28) one sees that the "rate" at which hops of length $r$ occur it is equal to $e^{-\lambda r}$ while the rate at which the packet passes them is $e^{-\lambda r p D_{1}(p)}$. Thus, when $p D_{1}(p)>1$ the packet delay becomes infinite.

\section{Density of progress}

Some MANET models (in particular the bipolar one) use the mean density of progress $d$ to optimize the performance of the model with respect to $p$ (cf e.g. [8]). Recall that in a 2D MANET $d$ is defined as the expected total progress of all the successful transmissions per unit of surface. In our 1D model, by Campbell's formula $d$ can be expressed as:

$$
d=p(1-p) \mathbf{E}^{0}\left[\left|X_{1}\right| \mathbb{I}\left(\operatorname{SINR}_{\left(0, X_{1}\right)} \geq T\right)\right] .
$$

The density of progress, can be also seen as quantifying the number of bit-(or packet)-meters "pumped" per unit length of a route.

Proposition 4.8: Under the assumptions of Proposition 4.1 the density of progress in the $N N$ receiver model is equal to:

$$
d=d_{N N}(p)=\frac{p(1-p)}{\left(1+p \mathcal{C}_{1}\right)^{2}}
$$

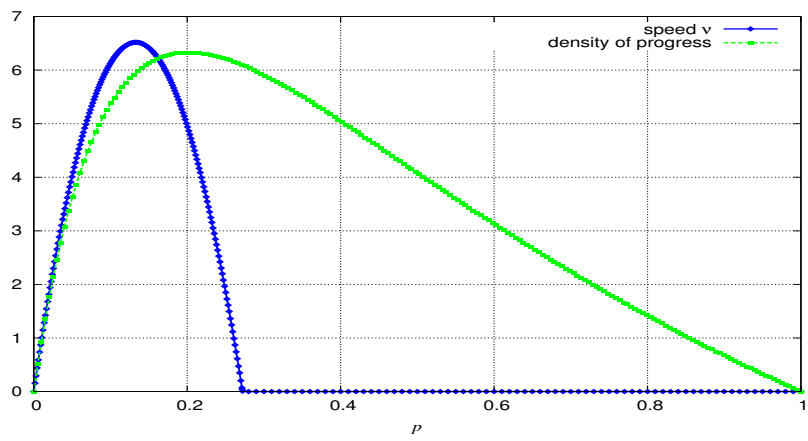

Fig. 2. Comparison of mean speed in the NN model and density of progress (both expressed in meters per slot duration); cf Section II-G and Example 4.6.

and is maximized for $p$ equal to:

$$
p_{N N}^{*}=\frac{\mathcal{C}_{1}+1-\sqrt{\mathcal{C}_{1}^{2}-1}}{2 \mathcal{C}_{1}} .
$$

Moreover, $0<p_{N N}^{*}<1$.

Proof: Using Campbell's formula, the density of progress in the NN model can be expressed as

$$
d_{N N}(p)=\lambda p(1-p) \mathbf{E}^{0}\left[R \mathbf{P}\left\{\mathbb{I}\left(F \geq T I_{\Phi^{1}} l(r) \mid R=r\right\}\right]\right.
$$

with the notation as in the proof of Proposition 4.1. Following the same arguments as in this latter proof we obtain:

$$
d_{N N}(p)=\lambda^{2} p(1-p) \int_{0}^{\infty} r e^{-\lambda r\left(1+p \mathcal{C}_{1}\right)} \mathrm{d} r,
$$

which is equal to the right-hand side of (4.32). Taking the derivative of the latter expression in $p$ we find that its sign is equal to that of the polynomial $P(p)=1-p\left(2+\mathcal{C}_{1}\right)-2 p^{2} \mathcal{C}_{1}^{2}+$ $2 p^{3} \mathcal{C}_{1}^{2}$. Note that $P(-\infty)=-\infty, P(0)=1, P(1)=-1-$ $\mathcal{C}_{1}<0$ and $P(\infty)=\infty$. Hence in the interval $(0,1), P(\cdot)$ has a unique root: $p_{N N}^{*}$ which maximizes $d_{N N}(p)$. The explicit expression for this root follows from the general formulas for the roots of cubic equations.

Remark 4.9: Note that density of progress is a "static" quantity, calculated with respect to one slot. It can also be easily evaluated for the NR model in contrast to the mean local delay. However, it fails to discover the existence of the critical value of $p$ for the performance of the MANET revealed by the analysis of the local delay and packet progression speed. Also, one may be tempted to approximate this speed by $d / \lambda$. Let us note, however, in Figure 2, that the optimization of this quantity in $p$ cannot be directly related to the maximization of the packet progression speed. For this reason we will not consider the density of progress any further in this paper.

\section{E. Mean end-to-end delay and speed of packet progression on finite segments of routes}

In this section we will study the issues of delays and the speed of packet progression on finite segments of routes. In this regard, we assume that in addition to the node located at the origin $X=0$ (also called $O$ ), a second node, being the final destination of a given packet is located at a fixed position $M>0$ on the line. Mathematically, this corresponds to the Palm distribution $\mathbf{P}^{0 M}$ of the Poisson pp, given these two fixed 
points. We want to compute the mean end-to-end delay for a given packet to leave the node 0 and reach node $M$ following NN routing. The situation proposed is shown in Figure 3. We denote this end-to-end delay by $L_{0 M}=\sum_{X_{i} \in[0, M)} D_{i}$.

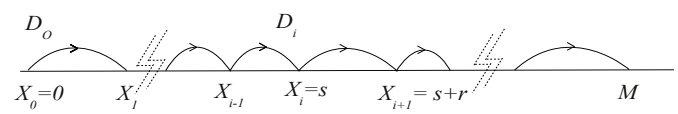

Fig. 3. Transmission delay from node 0 to node $M$.

Proposition 4.10: Under the assumptions of Proposition 4.1 the mean end-to-end delay in the NN routing on the distance $M$ is equal to

$$
\begin{aligned}
\mathbf{E}^{0 M}\left[L_{0 M}\right] & \frac{1}{p(1-p)}\left(e^{-\lambda M} E(M)\right. \\
& +\int_{0}^{M} \lambda e^{-\lambda r} E(r) G_{M}(0, r) \mathrm{d} r \\
& +\lambda \int_{0}^{M} \int_{0}^{M-s} E(r) G_{0}(s, r) G_{M}(s, r) \lambda e^{-\lambda r} \mathrm{~d} r \mathrm{~d} s \\
& \left.+\lambda \int_{0}^{M} E(M-s) G_{0}(s, M-s) e^{-\lambda(M-s)} \mathrm{d} s\right)
\end{aligned}
$$

with $E(r)=e^{\lambda p r \mathcal{D}_{1}(p)}, G_{0}(s, r)=h(s+r, r)^{-1}$ and $G_{M}(s, r)=h(M-s-r, r)^{-1}$.

Proof: The mean sum of the delays from the source node 0 to the destination node $X=M$ under $\mathbf{E}^{0 M}$ can be expressed using Campbell's formula as

$$
\mathbf{E}^{0 M}\left[L_{0 M}\right]=\mathbf{E}^{0 M}[D(0)]+\lambda \int_{0}^{M} \mathbf{E}^{0 M s}[D(s)] \mathrm{d} s,
$$

where the first term corresponds to the average exit time from node 0 and $\mathbf{E}^{0 M s}[D(s)]$ is the average exit time from a "current" node located at $s \in(0, M)$. These two expectations differ from $\mathbf{E}^{0}\left[L_{0}\right]$, expressed in (4.31), because there is a fixed node at $M$ which acts as an additional interferer but which also limits the hop length. Moreover, for the transmission from the current node $s \in(0, M)$, the node at 0 also acts as an additional interferer. We will show how the terms in (a)-(d) of (4.33) reflect these circumstances. Let us first remark from (4.31) that the function $E(r)$ is the expected exit time from a typical node, given its receiver is located at the distance $r$ and given no additional (non-Poisson) interfering nodes. Now, it is easy to conclude that the term (a) gives the expected end-to-end delay when it is necessary to make the direct hop from 0 to $M$ (when there is no Poisson relay node between them). The term (b) gives the expected exit time from 0 to its nearest receiver when it is located at some $r \in(0, M)$ :

$$
\begin{aligned}
& \mathbf{E}^{0 M}[D(0) ; r<M] \\
& =\frac{1}{p(1-p)} \int_{0}^{M} \lambda e^{-\lambda r} e^{\lambda p t \mathcal{D}_{1}(p)} h(M-r, r)^{-1} \mathrm{~d} r,
\end{aligned}
$$

where the factor $h(M-r, r)^{-1}=G_{M}(0, r)$ (not present in (4.31)) is due to the fact that $M$ acts as an additional interferer for the transmission from 0 to $r<M$. Similarly, the term (d) corresponds to the direct hop from the running

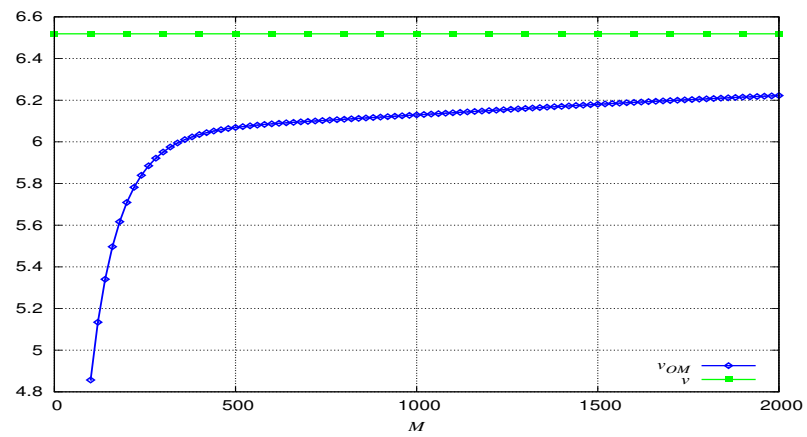

Fig. 4. Mean short-distance speed $v_{O M}$ with respect to $M$ (expressed in meters) in the $\mathrm{NN}$ model and long-distance speed $v$ (expressed in meters per slot duration); cf Section II-G.

node at $s$ to $M$ (when there is no Poisson relay node between them) and term (c) corresponds to the delay of going from $s$ to its nearest receiver $r \in(s, M)$. The node at 0 interferes with both these transmissions, which is reflected by the factors $G_{0}(s, M-s)$ and $G_{0}(s, r)$ in the terms (d) and (c), respectively. The node at $M$ also interferes with the transmission from $s$ to $r \in(s, M)$, whence $G_{M}(s, r)$ in (c).

Define the average speed of the packet progression on distance $M$ as

$$
v_{0 M}:=M / \mathbf{E}^{0 M}\left[\sum_{X_{i} \in[0, M)} D_{i}\right]=M / \mathbf{E}^{0 M}\left[L_{0 M}\right] .
$$

Proposition 4.10 allows one to express this quantity explicitly. ${ }^{6}$

Corollary 4.11: The average speed of the packet progression on distance $M$ is equal to $v_{0 M}=M / \mathbf{E}^{0 M}\left[L_{0 M}\right]$ where $\mathbf{E}^{0 M}\left[L_{0 M}\right]$ is given by (4.33).

Example 4.12: In Figure 4 we present the speed of the packet progression on distance $M$ assuming $T=10$ and $\beta=4$, for $M$ varying from 100 to 2000 . The calculation is performed for the value of $p$ that maximizes the asymptotic long-distance speed calculated in Proposition 4.5, presented as the horizontal line in Figure 4. The existence of the destination node in a finite horizon has a double impact on the packet progression. On the one hand it "attracts" the packet reducing the negative impact of long hops (cf Remark 4.7). Indeed, no hop can be longer than the direct hop to $M$ and thus the speed of packet progression is non-null even if $p$ is larger than the critical value for the long-distance speed, cf. Figure 2. On the other hand, it "repels" the packet, because it creates an additional interference, which is more significant when the packet is close to the destination, cf Figure 4 with $M$ from 0 to $250 \mathrm{~m}$ where the speed rapidly increases with the distance $M$ to the destination. One can conclude that routing on distances

\footnotetext{
${ }^{6}$ Another way of defining the average speed of the packet progression on distance $M$ consists in taking the expectation of the ratio $\mathbf{E}^{0 M}\left[M / L_{0 M}\right]$. Note (by the Jensen's inequality) that this latter choice gives larger values of the speed, but both are asymptotically equivalent in the sense that, as $M \rightarrow \infty$, they approach the mean long-distance speed $v$ considered is Proposition 4.10. The proposed definition has an advantage of being more tractable. It is also analogous to the usual definition of the mean user throughput describing the average speed of data transfer in communication networks, which is the average number of bits sent (or received) per data request to the average duration of the data transfer.
} 
shorter than some critical value $(M<250 \mathrm{~m})$ is inefficient in terms of exploiting of the route transport capacity.

\section{IMPACT OF EXTERNAL NOISE AND INTERFERENCE}

In the previous section we assumed that the external noise is negligible $(W=0)$. In this section we study the impact of a non-null external noise field $W^{n}(y)$ as in (2.2). As in Section III, we assume that the nodes of $\Psi$ use Aloha MAC with probability $p^{\prime}$. Recall also that the external noise $W$ is constant over time and independent both from the route $\Phi$ and the interfering nodes $\Psi$. We denote

$$
B(r)=e^{T W(A r)^{\beta}} \quad \text { and } \quad B^{-1}(r)=e^{-T W(A r)^{\beta}} .
$$

\section{A. Capture probability}

It is easy to extend the results of Propositions 4.1 and 4.8 to the case of an arbitrary noise field $W^{n}(y)$ as in (2.2).

Proposition 5.1: The probability of successful transmission by a typical node of Poisson route $\mathcal{R}=\Phi$ authorized by Aloha to transmit to its relay node in the NN routing model with noise and interference field $W^{n}(y)$ as in (2.2) is equal to

$$
p_{N N}=\lambda(1-p) \int_{0}^{\infty} e^{-r \lambda\left(1+p \mathcal{C}_{1}\right)} B^{-1}(r) \mathcal{L}_{\Psi}^{-H^{\prime}}(r) \mathrm{d} r .
$$

The expressions for $\mathcal{L}_{\Psi}^{-H^{\prime}}(r)$ in the case of Poisson $(P)$ and Poisson-line (PL) interferers are given in Lemma 3.4.

Proof: The proof follows the same lines as in the proof of Proposition 4.1, with the averaged noise factor $B^{-1}(r)=$ $\mathcal{L}_{W(r)}\left(T(A r)^{\beta}\right)$ and the averaged impact of interfering field captured by $\mathcal{L}_{\Psi}^{-H^{\prime}}(r)$; cf. Lemma 3.1 and expression (3.13).

\section{B. Long routes}

The following results show a very negative impact of arbitrarily small noise on the performance of $\mathrm{NN}$ routing on long routes.

Proposition 5.2: The mean local delay in the Poisson $\mathcal{R}=$ $\Phi N N$ routing is infinite, $\mathbf{E}^{0}\left[L_{0}\right]=\infty$, provided the noise is non-null noise, $\operatorname{Pr}(W>w) \geq \epsilon$ for some $w, \epsilon>0$, or the field of interfering nodes is super-Poisson in the sense of having Laplace transforms of negative functions not smaller than a Poisson point process (as e.g. Poisson-line $\Psi$ ) and applying Aloha with non-null MAP $p^{\prime}>0$. In this case the speed of packet progression on long routes is almost surely null.

Proof: As in the proof of proposition 4.3, cf. also Lemma 3.3 and expression (3.14), we have

$$
\mathbf{E}^{0}\left[L_{0}\right]=\frac{\lambda}{p(1-p)} \int_{0}^{\infty} e^{-\lambda r} e^{\lambda p r \mathcal{D}_{1}(p)} B(r) \mathcal{L}_{\Psi}^{H^{\prime}}(r) \mathrm{d} r .
$$

By our assumption on $W, B(r) \geq \epsilon e^{T w(A r)^{\beta}}$ and the integral $\int_{0}^{\infty} e^{-\lambda r\left(1-p \mathcal{D}_{1}(p)\right)+T w(A r)^{\beta}} \mathrm{d} r$ diverges for all $p>0$ since $\beta>2$. Regarding the impact of $\Psi$, we consider first the Poisson case. By (3.19), $\mathcal{L}_{\Psi}^{H^{\prime}}(r) \sim \exp \left[r^{2} p^{\prime} /\left(1-p^{\prime}\right)^{1-2 / \beta} \times\right.$ Const] and consequently $\int_{0}^{\infty} e^{-\lambda r\left(1-p \mathcal{D}_{1}(p)\right)} \mathcal{L}_{\Psi}^{H^{\prime}}(r) \mathrm{d} r$ diverges for all $p, p^{\prime}>0$. By the comparison to the Poisson case, this integral is divergent for point processes $\Psi$ which have larger Laplace transforms of negative functions.

Recall from Remark 3.6 that larger Laplace transforms indicate more clustering in node patterns.

Remark 5.3: The reason why the long distance speed is 0 lies in the fact that the hops in a Poisson route can be arbitrarily long and that such hops slow down (to zero) packet progression in the presence of noise. In order to understand why even a very small noise makes the mean local delay infinite, whereas the in-route interference does not (for suitable small MAC $p$ ) let us observe that this interference (at a given receiver) varies over time because the MAC decisions and fading variables are independent from slot to slot. Thus, even on a long hop, the packet has a chance to be transmitted when the in-route interference happens to be relatively small. In contrast, the noise is constant (not re-sampled) from slot to slot. This makes the fundamental difference, as already observed in [2]. Note also that external interference has the same dramatic impact as noise, even if it also varies over time slots. The explanation lies in the 2D geometry of the interfering field compared to $1 \mathrm{D}$ geometry of the route. More precisely, the probability of having the nearest in-line interferer within a distance larger than $r$ is of order $e^{-r}$, while the analogous probability regarding the nearest interferer on the plane is of order $e^{-r^{2}}$ for large $r$. This probability is too small to let the packet traverse a long nearest-neighbour hop on the line, whose probability scales as $e^{-r}$. Let us recall from [2] that the mean local delay of the transmission to the nearest neighbour in 2D Poisson MANETs (without noise) is finite for sufficiently small $p$, since in this case both the hop-distance and the nearest interferer distance scale as $e^{-r^{2}}$.

One method of coping with overly long hops consists in adding "fixed" points to the Poisson route $\mathcal{R}$. In fact, we will show in Section V-C that adding only the fixed destination node $M$ is sufficient to make the end-to-end delay finite in the presence of noise. For infinite routes one needs, however, a "fixed" regular grid of relaying nodes; cf Section V-D.

\section{Short routes}

Our setting is now as in Section IV-E, i.e., we consider the Poisson route $\Phi$ under Palm with fixed origin and destination nodes 0 and $M$, and we compute the end-to-end delay assuming some external noise $W$ and a field of the form created by a stationary pattern of interfering nodes $\Psi$.

Proposition 5.4: Under the assumptions of Proposition 4.1 and in the presence of the external noise of the form (2.2), as explained above, the mean end-to-end delay from 0 to $M$ in Poisson NN routing is given by (4.33) with $E(\cdot)$ (given in Proposition 4.10) replaced by $E(\cdot) \times B(\cdot) \times \mathcal{L}_{\Psi}^{H^{\prime}}(\cdot)$, with $B(\cdot)$ given in (5.34). The expressions for $\mathcal{L}_{\Psi}^{H^{\prime}}(\cdot)$ in the case of Poisson $(P)$ and Poisson-line $(P L)$ interferers are given in Lemma 3.4.

Proof: Observe first that the conditional capture probability given the receiver in $\Phi$ at distance $r$, and given the interfering pattern $\Psi$, which we denote by $\pi(r, \Phi, \Psi)$, is equal 
to

$$
\begin{aligned}
\pi(r, \Phi & , \Psi) \\
= & (1-p) \exp \left\{\sum_{X_{i} \in \Phi} \log \left(1-\frac{p}{1 / T\left(\left|X_{i}\right| / r\right)^{\beta}+1}\right)\right\} \\
& \times \exp \left\{\sum_{Y_{i} \in \Psi} \log \left(1-\frac{p^{\prime}}{1 / T\left(\left|Y_{i}\right| / r\right)^{\beta}+1}\right)\right\} \\
& \times \exp \left(-T W(A r)^{\beta}\right) ;
\end{aligned}
$$

cf the proof of Proposition 4.3. Hence, by independence, the mean local delay, given the distance to the receiver $R=r$, is equal to

$$
\frac{1}{p} \mathbf{E}^{0}\left[\frac{1}{\pi(r, \Phi, \Psi)} \mid R=r\right]=\frac{E(r) \mathcal{L}_{\Psi}^{H^{\prime}}(r) B(r)}{p(1-p)} .
$$

The remaining part of the proof follows the same lines as the proof of Proposition 4.10.

Remark 5.5: (Interferers' clustering paradox) An immediate consequence of Corollary 3.5 is that both coverage probability $p_{N N}$ and the mean end-to-end delay $\mathbf{E}^{0 M}\left[L_{0 M}\right]$ are larger in the model with Poisson-line field of interferers than in the homogeneous Poisson field having the same spatial intensities of points $\mu=\lambda^{\prime} \nu$. Recall that this apparent paradox can be explained by noting that when forwarding packets in the clustering field of interferers one has to "traverse" the regions where interfering nodes have a higher than average density. This can considerably slow down packet propagation, and make it lose the advantage gained in regions with a smaller density of interferers. The degradation increases with the degree of clustering (cf [5, 6]) and with routing distance.

Example 5.6: The average speed of the packet progression on the distance from $O$ to $M$ can be expressed as in Corollary 4.10. We now present some numerical illustrations how this speed depends on the model's parameters. We first assume only constant noise $W$. Figure 5 shows the speed of packet progression with respect to $W^{7}$. We have assumed $T=10$, $\beta=4$ and $p=0.15$, the value which maximizes the average long-distance speed without noise. The curves correspond to different routing distances $M=100,1000,10000$. If we expect that an emergency packet (DENM) be transmitted in less than $0.2 \mathrm{~s}$ over $1 \mathrm{~km}$, we must have $W \leq-153 \mathrm{~dB}$ for $M=10000, W \leq-123 \mathrm{~dB}$ for $M=1000$. It is impossible for $M=100$. Figure 6 shows the same speed of packet progression with respect to $M$ for $W=-110,-130,-150 \mathrm{~dB}$. For the various values of $M$ we keep $p=0.15$ the value which maximizes the average long-distance speed without noise. If we expect an emergency delay smaller than $0.2 \mathrm{~s}$ over $1 \mathrm{~km}$, we must have $M \in[120,350]$ for $W=-110 \mathrm{~dB}, M \in[110,780]$ for $W=-120 \mathrm{~dB}$ and $M \in[110,1770]$ for $W=-130 \mathrm{~dB}$.

Both Figures 5 and 6 show a sharp cut of phenomenon: the speed of packet progression is approaching zero beyond some critical noise level and/or route length. The impact of the noise is stable below its critical value (for a given distance). Comparing Figure 4 to Figure 6 we see that after the "fast acceleration" the packet reaches a constant speed which can

\footnotetext{
${ }^{7} \mathrm{~W}$ is expressed in $\mathrm{dB}$ according to the formula $10 \log _{10}(W / P)$ where $\mathrm{P}$ is the actually transmitted power which we suppose to be 1 .
}

be maintained on routes shorter than some critical length depending on the level of noise.

Figure 7 shows the mean end-to-end speed with respect to the spatial density of the field of Poisson interferers $\lambda^{\prime}$ for two values of the Aloha parameter $p^{\prime}=0.15$ and $p^{\prime}=0.015$ used by the interferers. As in the case of constant noise, the speed rapidly falls from an almost constant value (corresponding to the case without interferers) to zero. Figure 8 shows the mean end-to-end speed with respect to the spatial density of the field of the Poisson-line process of rate $\nu$ and $\lambda^{\prime}$ for $p^{\prime}=0.15$ and $p^{\prime}=0.015$. We observe that this speed is smaller than the mean speed in a field of Poisson interferers with a comparable spatial density $\nu \times \lambda^{\prime}$ (cf. Figure 7); this confirms remark 3.5. For $p^{\prime}=0.15$, an emergency delay over $1 \mathrm{~km}$ smaller than $0.2 \mathrm{~s}$ is achieved for $\lambda^{\prime} \leq 10^{-6.7}$ in a Poisson field and for $\nu \lambda^{\prime} \leq 10^{-7.09}$ in a Poisson process of lines.

\section{Equipping long Poisson routes with fixed relay stations}

As previously mentioned, in order to solve the problem of long hops slowing down the packet progression in the presence of external noise, one can add fixed relay stations, thus allowing efficient routing on long Poisson routes. In this section we will study this problem.

We assume regularly spaced "fixed" relay nodes $\mathcal{G}=$ $\left\{n \Delta+U_{\Delta}, n \in \mathbb{Z}\right\}$, where $\Delta>0$ is some fixed distance. These nodes are added to the Poisson route $\Phi$, they use the same Aloha parameter $p$ and the NN routing is considered on $\Phi \cup \mathcal{G}$.

We wish to compute the mean local delay on $\Phi \cup \mathcal{G}$ in the presence of noise, and thus characterise the speed of packet progression on long routes equipped with fixed relay stations. This will allow us to optimise the performance of the network in the parameter $\Delta$. It should be noted that a small value of $\Delta$ makes the fixed relay nodes too dense, creating a lot of interference and making the routing hops very small. On the other hand, a large value of $\Delta$ leads to long hops which slow down the packet progression.

Proposition 5.7: Assume $\mathcal{R}=\Phi \cup \mathcal{G}$ to be a Poisson route equipped with fixed relay stations with the inter-relay distance $\Delta$, as described above. The mean local delay in the NN routing on this model in the presence of external noise of the form (2.2) as in Proposition 5.4 is equal to:

$$
\begin{aligned}
& \mathbf{E}^{0}[L] \\
& =\frac{\lambda}{p(1-p)(\epsilon+\lambda)}\left(\frac{1}{\Delta} \int_{0}^{\Delta} E(z) E^{\prime}(z) e^{-\lambda z} e^{H(0, z)} \mathrm{d} z\right. \\
& \left.+\frac{\lambda}{\Delta} \int_{0}^{\Delta} \int_{0}^{z} E(r) E^{\prime}(z) e^{-\lambda r} e^{H(z-r, r)+\log (h(z-r, r))} \mathrm{d} r \mathrm{~d} z\right) \\
& +\frac{\epsilon}{p(1-p)(\epsilon+\lambda)}\left(\frac{\lambda}{\Delta} \int_{0}^{\Delta} E(z) E^{\prime}(z) e^{-\lambda z} e^{H(-z, z)} \mathrm{d} z\right. \\
& \left.\quad+e^{-\lambda \Delta} B(\Delta) E(\Delta) e^{H(0, \Delta)-\log (h(\Delta, \Delta))}\right)
\end{aligned}
$$

with $\epsilon=\frac{1}{\Delta}$ and $H(z, r)=\sum_{n \in \mathbb{Z}, n \neq 0} \log (h(n \Delta+z, r))$.

Proof: Let us suppose that a typical node 0 of $\Psi \cup \mathcal{G}$ is at $X=0$. We know that this typical node belongs to $\Psi$ with 


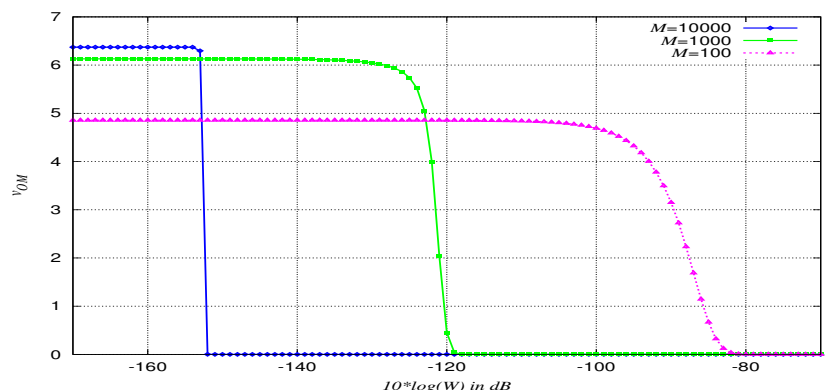

Fig. 5. Mean short-distance speed (in meters per slot duration) with respect to noise $W$ in the NN model.

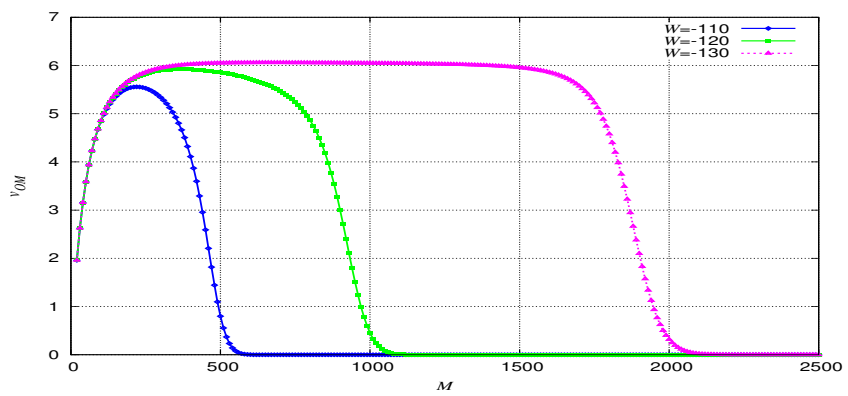

Fig. 6. Mean short-distance speed (in meters per slot duration) with respect to the distance $M$ (in meters) in the NN model, $W=-110,-120,-130 \mathrm{~dB}$.

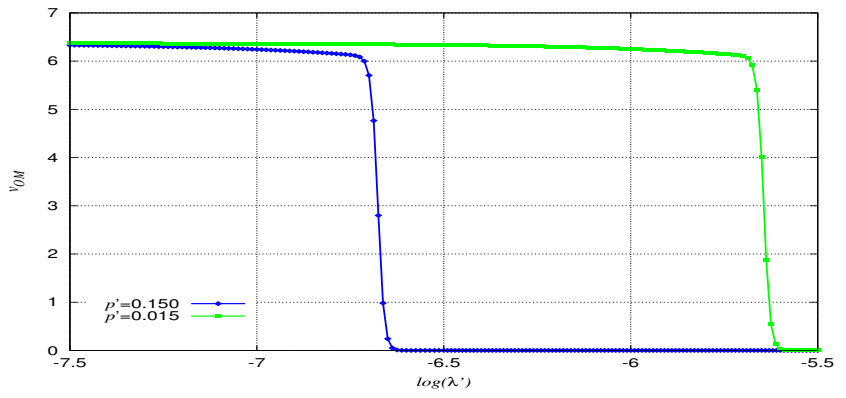

Fig. 7. Mean short-distance $(M=10000 \mathrm{~m})$ speed (in meters per slot duration) in the Poisson field of interferers with respect to it spatial intensity $\lambda^{\prime}\left(\right.$ nodes per $\left.\mathrm{m}^{2}\right)$.

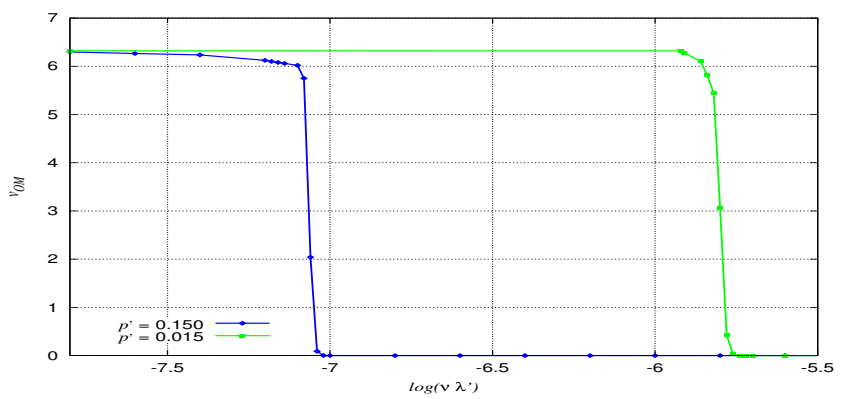

Fig. 8. Mean short-distance $(M=10000 \mathrm{~m})$ speed (in meters per slot duration) in the Poisson-line of interferers, in with respect to the spatial intensity of nodes $\nu \lambda^{\prime}$ (nodes per $\mathrm{m}^{2}$ ); $M=10000 \mathrm{~m}$. To be compared to the Poisson case on Figure 7.

probability $\frac{\lambda}{\epsilon+\lambda}$ and to $\mathcal{G}$ with probability $\frac{\epsilon}{\epsilon+\lambda}$. Thus we have

$$
\mathbf{E}^{0}[L]=\frac{\lambda}{\epsilon+\lambda} \mathbf{E}^{0}[L \mid 0 \in \Psi]+\frac{\epsilon}{\epsilon+\lambda} \mathbf{E}^{0}[L \mid 0 \in \mathcal{G}] .
$$

Let us now assume that the typical node 0 is in $\Psi$ and we use the fact that the nodes in $\Psi$ are independent of the nodes in $\mathcal{G}$.

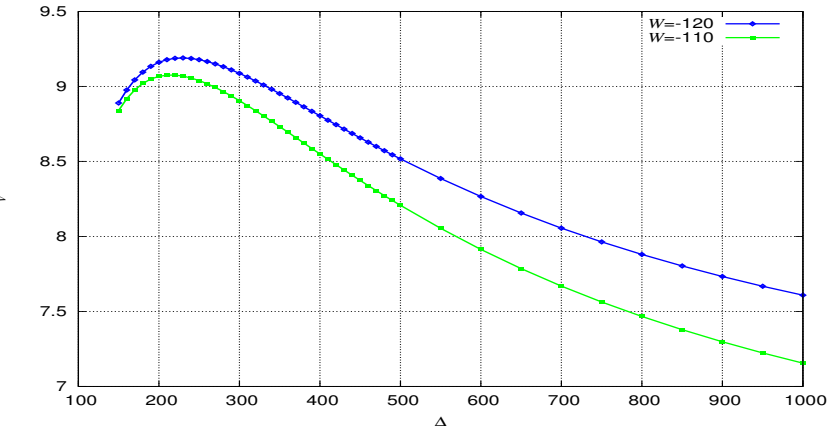

Fig. 9. Mean long-distance speed (in meters per slot duration) with respect to $\Delta$ (in meters) for $W=-110 \mathrm{~dB}$ and $W=-120 \mathrm{~dB}$.

If its closest node (towards the right) is in $\mathcal{G}$, the average delay to leave node 0 is : $B(z) E(z) e^{H(0, z)}$. Conditioning by the fact there is no node of $\Psi$ in $[0, z]$ (that occurs with probability $e^{-\lambda z}$ ), we find the first integral in the first line of (5.36). We still assume that the typical node is in $\Psi$ but now we also assume that the closest node (towards the right) is in $\Psi$. Computing the average delay in this case, we find the second double integral of the first line of (5.36); $\lambda e^{-\lambda r}$ is actually the density of the right-hand neighbor's position. The contribution $e^{\log (h(z-r, r))}$ corresponds to the fact that the node in $\mathcal{G}$ located at $z$ is not taken into account by $e^{H(z-r, r)}$ since the summation in $H$ is for $n \neq 0$.

The second part of the contribution for $\mathbf{E}^{0}[L]$ corresponds to the case where node 0 is in $\mathcal{G}$. If we assume that the righthand neighbor of 0 is in $\Psi$, the contribution of the delay corresponds to the integral of the proposition in the second line of $\mathbf{E}^{0}[L]$. There is no correction needed for $H(-z, z)$ since the typical node 0 is in $\mathcal{G}$ and does not contribute to the delay as it is in $H(-z, z)$. The last term of the second line in (5.36) corresponds to the case where node 0 and its righthand neighbor are in $\mathcal{G}$. This occurs with probability $e^{-\lambda \Delta}$. The node in $\mathcal{G}$ located at $\Delta$ does not contribute to the delay since we analyze the delay from 0 and its right-hand neighbor in $\mathcal{G}$ (thus located at $\Delta$ ). Thus $H(0, \Delta)$ must be corrected by $e^{-\log (h(\Delta, \Delta))}$.

Example 5.8: In Figure 9, we plot the mean long-distance speed $v=1 /\left(\lambda \mathbf{E}^{0}[L]\right)$ with respect to $\Delta$ with fixed noise of levels $W=-110 \mathrm{~dB}$ and $W=-120 \mathrm{~dB}$. Comparing Figure 6 to Figure 9 one can observe that the optimal interrelay distance $\Delta$ of the fixed structure corresponds to the smallest length $M$ of the route on which the mean speed of packet progression attains its maximum without the fixed infrastructure. In other words, the relay stations should "break down" long routes into segments of the length optimal for the end-to-end routing given the noise level. The emergency delay for $1 \mathrm{~km}$ is $0.103 \mathrm{~s}$ for $W=-110 \mathrm{~dB}$ and 0.108 for $W=-120 \mathrm{~dB}$.

\section{CONCLUding REMARKS}

We have studied performances of end-to-end routing in MANETs, using a linear nearest-neighbor routing model embedded in an independent planar field of interfering nodes using Aloha MAC. We have developed numerically tractable expressions for several performance metrics such as the endto-end delay and speed of packet progression. They show 
TABLE I

Mean Propagation SPeEd in a linear Poisson network with Aloha MAP $p$.

\begin{tabular}{|l|l|l|l|}
\hline given distance $M$ & $\begin{array}{l}\text { external noise or super- } \\
\text { Poisson interference field }\end{array}$ & adding fixed relays \\
& $\begin{array}{l}\text { rapidly increasing with } \\
M \text { and stabilizing or } \\
\text { decreasing back to } 0 \\
\text { given interval, approach- } \\
\text { ing zero for } M \text { outside } \\
\text { for large } M \text { depending } \\
\text { whether } p<p_{\text {critical }} \text { or }\end{array}$ & $\begin{array}{l}\text { increasing with } M \text { and } \\
\text { stabilizing for large } M\end{array}$ \\
& not & & \\
\hline asymptotically $M \rightarrow \infty$ & positive provided & zero & positive for all $p$ \\
\hline
\end{tabular}

super-Poisson field - a field clustering more than Poisson in the sense of having larger Laplace transforms of negative functions

$p_{\text {critical }}=\sup \left\{p: p \mathcal{D}_{1}(p)<1,0 \leq p \leq 1\right\}$

TABLE II

CLUSTERING PARADOX; COMPARISON OF THE IMPACT OF THE EXTERNAL FIELD OF INTERFERING NODES.

\begin{tabular}{|l|c|c|}
\cline { 2 - 3 } \multicolumn{1}{c|}{} & $\begin{array}{c}\text { Poisson field } \\
\text { (more homogeneous) }\end{array}$ & $\begin{array}{c}\text { Poisson-line field } \\
\text { (more clustering) }\end{array}$ \\
\hline capture probability & \multicolumn{2}{|c|}{$<$} \\
\hline propagation speed & \multicolumn{2}{|c|}{$>$} \\
\hline
\end{tabular}

how network performance can be optimized by tuning Aloha and routing parameters. In particular, we show a need for a well-tuned lattice structure of wireless relaying nodes (not back-boned) which help to relay packets on long random routes in the presence of a non-negligible noise. We have also proved and explained why for a given intensity of interferers, the propagation speed is higher when the interferers are more homogeneously distributed, even if, paradoxically, the probability of a single successful transmission is smaller in this case. Numerical comparisons in this regard have been shown for Poisson and Poisson-line processes. The qualitative summary of the results is presented in Tables I and II.

The strength of the present paper is that it offers exact, closed-form expressions of the performance metrics considered. This raises the question of how far this rigorous approach can be extended.

Although our analysis does not take into account packet queuing at the relay nodes, it covers the practical case of broadcasting of emergency packets, which are transmitted in priority at each relay. Our developments use Aloha rather than a CSMA protocol, which is generally more efficient than Aloha. Thus, our results can be considered as conservative bounds regarding real VANETs with a CSMA protocol.

To handle CSMA protocols, approximations will be certainly necessary since, in contrast to Aloha, the transmission by one node depends on the activity of other nodes. Packet queuing further strengthens this dependence. The paper does not consider mobile nodes, nor nodes leaving the network, which could be a more realistic assumption regarding VANETs.

\section{REFERENCES}

[1] F. Baccelli and B. Błaszczyszyn. Stochastic Geometry and Wireless Networks, Volume II - Applications, volume 4, No 12 of Foundations and Trends in Networking. NoW Publishers, 2009.
[2] F. Baccelli and B. Błaszczyszyn. A new phase transition for local delays in MANETs. In Proc. of IEEE INFOCOM, San Diego CA, 2010.

[3] F. Baccelli, B. Błaszczyszyn, and O. Mirsadeghi. Optimal paths on the space-time SINR random graph. Adv. Appl. Probab., 43 (1):131-150, 2011.

[4] B. Błaszczyszyn and D. Yogeshwaran. Connectivity in subPoisson networks. In Proc. of 48 th Annual Allerton Conference, University of Illinois at Urbana-Champaign, IL, USA, 2010.

[5] B. Błaszczyszyn and D. Yogeshwaran. On comparison of clustering properties of point processes. Adv. Appl. Probab., 46(1):1-21, 2014.

[6] B. Błaszczyszyn and D. Yogeshwaran. Clustering comparison of point processes with applications to random geometric models. In V. Schmidt, editor, Stochastic Geometry, Spatial Statistics and Random Fields: Models and Algorithms, volume 2120 of Lecture Notes in Mathematics, chapter 2, pages 31-71. Springer, 2014.

[7] S.P. Weber, X. Yang, J.G. Andrews, and G. de Veciana. Transmission capacity of wireless ad hoc networks with outage constraints. IEEE Tran. Inf. Theory, 51(12):4091-4102, 2005.

[8] F. Baccelli, B. Błaszczyszyn, and P. Mühlethaler. An Aloha protocol for multihop mobile wireless networks. IEEE Trans. Inf. Theory, 52:421-436, 2006.

[9] F. Baccelli, B. Błaszczyszyn, and P. Mühlethaler. Stochastic analysis of spatial and opportunistic Aloha. IEEE JSAC, special issue on Stochastic Geometry and Random Graphs for Wireless Networks, 2009.

[10] M. Haenggi. Outage, local throughput, and capacity of random wireless networks. IEEE Trans. Wireless Comm., 8:4350-4359, 2009.

[11] M. Haenggi. The local delay in poisson networks. IEEE Trans. Inf. Theory, vol. 59:pp. 1788-1802, March 2013.

[12] Yi Zhong, Wenyi Zhang, and M. Haenggi. Managing interference correlation through random medium access. IEEE Trans. Wireless Comm., 13(2):928-941, February 2014.

[13] P. Gupta and P.R. Kumar. The capacity of wireless networks. IEEE Trans. on Information Theory, 46(2):388-404, mar 2000.

[14] Massimo Franceschetti, Olivier Dousse, David N. C. Tse, and Patrick Thiran. Closing the gap in the capacity of wireless networks via percolation theory. IEEE Trans. on Networking, 53(3):1009-1018, 2007.

[15] R.K. Ganti and M. Haenggi. Bounds on the information propagation delay in interference-limited aloha networks. In 
Proc. of WiOPT, Seul, 2009.

[16] P. Jacquet, B. Mans, and G. Rodolakis. Information propagation speed in mobile and delay tolerant networks. IEEE Tran. Inf. Theory, 56(10):5001-5015, 2010.

[17] K. Stamatiou and M. Haenggi. The delay-optimal number of hops in poisson multi-hop networks. In Proc. of ISIT, pages 1733-1737, 2010.

[18] K. Stamatiou and M. Haenggi. Delay Characterization of Multihop Transmission in a Poisson Field of Interference. IEEE/ACM Transactions on Networking, 2013.

[19] Alessandro Crismani, Udo Schilcher, Stavros Toumpis, Günther Brandner, and Christian Bettstetter. Packet travel times in wireless relay chains under spatially and temporally dependent interference, 2013.

[20] E. Baccelli, P. Jacquet, B. Mans, and G. Rodolakis. Highway vehicular delay tolerant networks: Information propagation speed properties. IEEE Tran. Inf. Theory, 58(3):1743-1756, 2012.

[21] H. Wu, R. Fujimoto, and G. Riley. Analytical models for information propagation in vehicle-to-vehicle networks. In Proc. of VTC2004-Fall, 2004.

[22] Z. Zhang, G. Mao, and B. Anderson. On information propagation speed in mobile vehicular ad hoc networks. In Proc. of Gobecom 2010, Miami, Florida, USA, 2010.

[23] Frédéric Morlot. A population model based on a Poisson line tessellation. In WiOpt 2012 - 10th International Symposium of Modeling and Optimization of Mobile, Ad Hoc, and Wireless Network, Paderborn, Germany, May 2012.

[24] D. J. Daley and D. Vere-Jones. An Introduction to the Theory of Point Mathematical Principles of Signal Processing Processes, vol. 2. Springer, 2008. 